\title{
Emergency Protection System for Women
}

\author{
S.Srikanth, G. Harish Kumar
}

\begin{abstract}
This venture depicts about a savvy keen security framework for ladies. Ladies everywhere throughout the world are confronting a lot of untrustworthy physical badgering. This procures a quick pace because of absence of a reasonable reconnaissance framework. Our task is a challenge to decide this issue. We are using two things wrist band and shows that are used in regular daily existence. A device takes after the group of a wrist got together by strain key at data impels show the end result shattering alert \& noxious gab instrument of a constrained self-opposite reason \& send region of a emergency message the contact and moreover comprehend enemy uses exist spouting tape. Nerve chat framework at a live spill capture using webcam is combined into a scenes going as an instrument to the insightful advancement. We genuinely acknowledge of endeavor will have any sort of impact on the female life.
\end{abstract}

Keywords: Women Security, ARMV7-A GSM, Raspberry pi 2, GPS, Streaming Video.

\section{INTRODUCTION}

The status of ladies in India has experienced particular stunning changes over the extent of the last barely any various years. In present day India, ladies prop up toward social issues and are as routinely as potential fiascos of misuse and savage encroachment and, as showed by a general assessment drove by Thomson Reuters, India is the "fourth most risky nation" on earth for ladies, and the most discernibly horrendous nation for ladies among the G20 nations. This undertaking turns around a security structure that is filtered through just to reasonably give security and flourishing to ladies so they never snatch acquainted while remaining with such social issues. The Delhi "Nirbhaya" case that set off the entire country was the best inspiration for this undertaking. It was high time we ladies required a change.

\section{EXISTING SYSTEM}

While proposing inspecting a similar concern particular fashioners have sorted out inventive applications. Major existing applications are-

\section{A. Application of Vith $U$ :}

These crisis uses in progress with a recognizable Indian horrible direct plan of TV "Gumrah" offer a control [V]. VithU, is a crisis App on the snap the power of catch of other Smart phone on various events interminably starts giving engineered messages at standard between times and contacts that you supply into the application as appointed beneficiaries or gatekeepers. The message sends the "I am in probability. I want assistance. You should scan for after my district."

\section{B.The immobilizer:}

This little weapon reproaches an attacker for an electric amaze. The smother disables the aggressor rapidly, giving you test opportunity to run away from the view. Right when the trigger is pull, an immobilizer manages around 700,000 volts into the assailant's body. Some immobilizers are satisfactorily little to be waged holy war of a cigarettes pack. Run on Lithium batteries \& can be passed in totes are detained in stomach area ties.

\section{Retaliate}

Retaliate application is a particularly focal application like ones recorded beginning at now. In any case, one uncommon part we like about the application is the Facebook notice. By giving Email \& SMS decisions an alert another individual throughout inconvenience, this application additionally restores Facebook your status.

\section{PROPOSED DESIGN}

This conventional structure plan a beneficial gadget will takes after band of wrist. This contains switch Pressure, GSM modem, Raspberry pi 2, GPS beneficiary, shattering alert, Live Steaming Video and Tear gas. Right where the switch Pressure is squashed, the gadget was induced typically by the unassuming amount on the milliseconds. Rapidly district of hurting the individual soughing past $\&$ messages sent by crisis contacts. The hollering prepared a unit prompted \& will pass on alert resonance $t$ will get down for help. Nerve chatter is applied to harm the assailant it will assist the hurt individual with escaping. Streaming Live Video will made to technique the circumstance of shocking misfortune utilizing a favored IP address will help us to separates a substance of aggressor close by the including condition of grasps feasibly.. Harmful gas discharge \& subsist gushing tape utilizing web cam are merged in the scenes go about as another weapon for sharp progression. 


\section{METHODOLOGY}

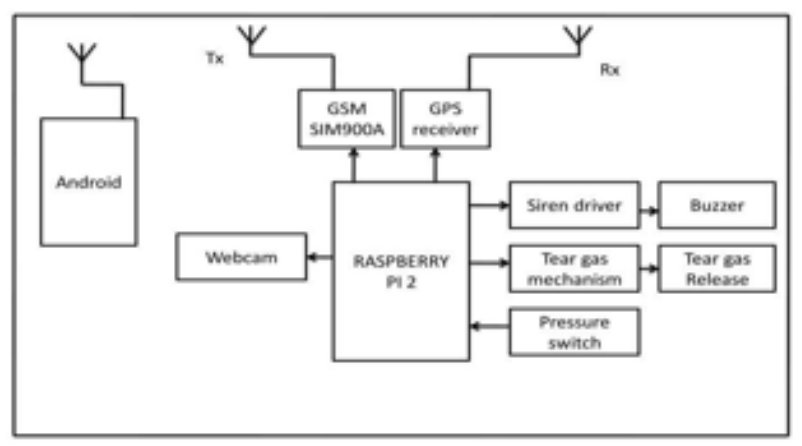

Figure: 1. Methodology the System

A. pi Raspberry

The Pi Raspberry is a headway of Master card reviewed single board PCs made in the United Kingdom with the Pi Raspberry base by the throbbing for moving of availability in the basic programming masterminding in making \&schools nations. The Raspberry Pi 2 depends in the Broad com BCM2836device of sever (So C), with the join of a ARMcortexv7 900mhz pc, Core Video IV GPU ,from the earliest starting point passed by the 256 megabytes of RAM. Starting at June 8, 2015, around 5 to 6,000,000 Pi's Raspberry has been sold. The Pi 2 Raspberry, were discharged in 2015 February.

\section{B. SIM900A GSM}

A modem of GSM has a specific kind of modem was like a works and SIM card above a joint effort of a adaptable chief, much comparable to a cell phone. From the flexible authority viewpoint, a GSM modem looks simply like a PDA. Right when a GSM modem is related with a PC, this attracts the PC to utilize the GSM modem to give over the adaptable structure. While these GSM modems are all around that truly matters now and again used to give flexible web sort out, huge proportions of them can other than be utilized for sending and enduring SMS and MMS messages. Adaptable affiliations based of GSM development has first pushed in 1991 in Finland. The SIM900A is a finished Dual-band GSM/GPRS module in a SMT type which is composed particularly for Chinese market, engaging you to profit by little estimations and financially smart frameworks.

Checking an industry-standard interface, the SIM900A passes on GSM/GPRS 900/1800MHz execution for voice, SMS, Data, and Fax in a little structure factor and with low power use. With a minor arrangement of $24 \mathrm{~mm} \times 24 \mathrm{~mm} \times 3 \mathrm{~mm}$, SIM900A can fit all around that genuinely matters of a necessities in the uses, particularly thin \& humbler enthusiasm of structure.

\section{Receiver of a GPS}

The Module of a GPS consistently getting data from the satellite and transmits correspondingly to the RS232. This made with the US division guardian (DOD).GPS indication is apply for radio wire commitment The module, $\&$ a full scale dynamic information by the position, speed $\&$ time data was appeared of a sequential line. When present date, longitude, scope, time, stature, travel \& speed course among the information, given with the module \& c utilized in a substitute uses including course, ocean control the officials, following structures, mapping and mechanical improvement.

This unit bolster up to 51 networks. GPS plan relates little structure factor contraptions which pass on fundamental degrees of progress in GPS introductions, exactness, bargain, enlisting force and adaptability. They are utilized to disentangle the installed structure bargain procedure.

\section{Streaming of a Live Video}

With structuring of a wifi, we obtain an internet protocol ad dress. Utilizing Log itech c270 web cam, with demonstrating a movement of a programming in pi 2 Raspberry model $\mathrm{B}$. We are seen the live gushing video or else we can spare the video for the record reason or catch the picture haker finally we put him after the bars.

\section{E. highlight of Extra}

An Electronics supplies 3V engine guide used for dangerous gab structure was utilized by the combined at one side of introductions. These should hurt enemy, where it is showered to eyes. For Alarm Screaming, are utilizing the electromagnetic $3 \mathrm{~V}$ sign get down for help out. Whole instruments worked when switch weight press end which create in the worst band.

\section{RESPONCE}

The Right switch weight is squashed, a sign turn-on. Shows was using with the individual being insinuated, of correct $50 \%$ of the aspects are will destructive gab should be sprinkled of the eyes of assailant to hurting a aggressor actually \& to the turnaround plane, the cam is constant to picture of a exist spilling record.

At the perpendicular section close to the alert will sent urgent contacts. The got individuals of which will open the internet protocol address fixed the urgent call list to seen the be alive video, so this will be valuable to get the correct address a motivator for the individual being suggested.

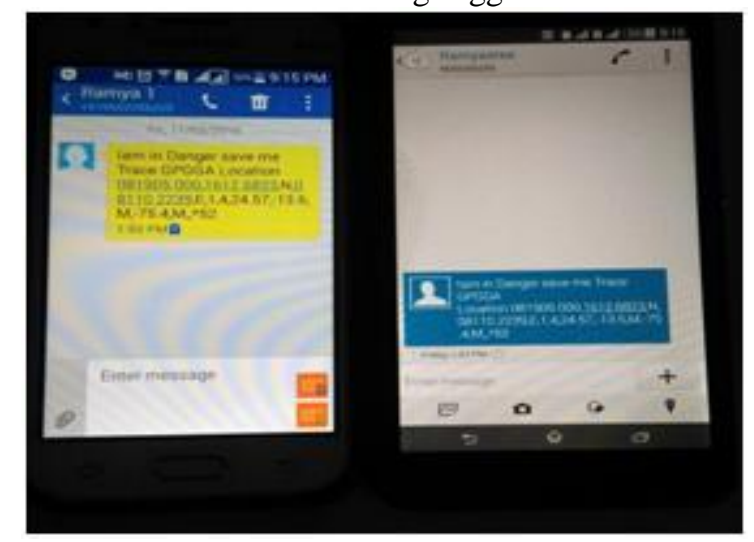

Figure: 2. Portray the area and the communication crisis will links.

The Diagram 2, shows the picture of an urgent associates the shown information "I'm in threat save me" nearer places of the existing GPS beneficiary. These caution of the urgent contacts will help us successfully the individual being referred to. 


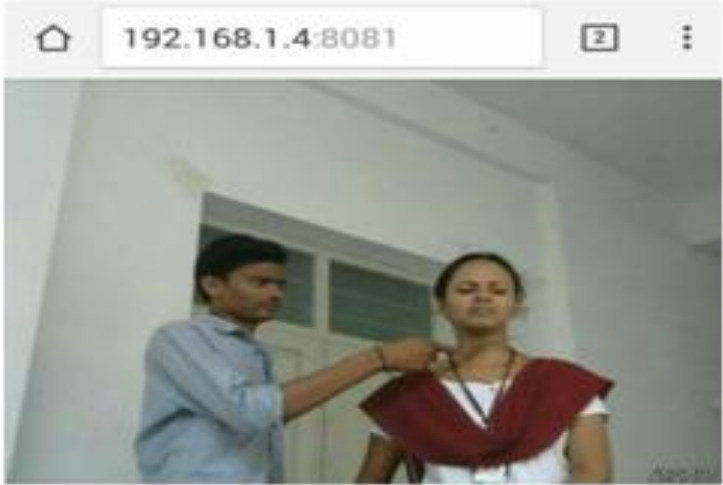

Figure: 3 Describes the Live stream Video of the Snapshot length of by internet protocol address

From Figure3, shows that the live spouting video image of gotten nearby internet protocol address. That is starting at now fix to the contacts list.

\section{CONCLUSION}

The paper shows organizing regarding the fundamental problems looked by women at this right days and will handle them precisely with diminished rigging and contemplations. Uses a wast limit \&displays, this section like noxious chatter revels, yelling alerts, live spouting tape \& disturbing the urgent contact with sends messages by the region. These device will beat fear that frenzies every female in the world about her security and prosperity

\section{REFERENCES}

1. Embedded systems by jack G. ganssle

2. Smart girls security system-Prof. Basavaraj Chougula, Archana Naik, Monika Monu, Priya Patil and Priyanka Das,International Journal of Application or Innovation in Engineering \& Management (IJAIEM) ISSN:2319-4847 Volume 3, Issue 4, April 2014

3. "electronic device for women safety"-Times of India, Sep 152013

4. Self defence system for women with location tracking and SMS alerting through GSM network-B.Vijaylashmi, Renuka.S, Pooja Chennur, Sharangowda. Patil International Journal of Research in Engineering and Technology(IJRET) eISSN: 2319-1163 | pISSN: 2321-7308 Volume: 04 Special Issue: 05

5. Reardon, op. cit., "Feminist Concepts of Peace and Security," p. 139

6. https://www.raspberrypi.org

7. G.Masario,M.Torchiano and M.Violante, An in-vehicle infotainment software architecture Based on Google Android, IEEE International Symposium on Industrial Embedded Systems 2009, 8-10 July 2009, pp. $257-260$

8. NMEAData. http://www.gpsinformation.org/dale/nmea.html.

9. ATcommands: http://www.develpershome.com/sms/atComandsIntro.asp

10. Dr. Aditi Jain and Ms. Shivani Gambhir. Socio-Economic Women Empowerment: Sharp Focus, International Journal of Advanced Research in management, 6(1), 2016, pp. 38-49.

11. D.Shanthi Revathi and Dr. Jayasree Krishnan. Problems and Opportunities of Women Entrepreneurs Faced in the Globalized Economy, International Journal of management, 3(1), 2012, pp. $77-81$

\section{AUTHORS PROFILE}

S.Srikanth, Asst. Professor

Dept. of ECE, MRECW, Secunderabad

G. Harish Kumar, Asst. Professor

Dept. of ECE, MRECW, Secunderabad 